\title{
A Discrete Numerical Scheme of Modified Leslie-Gower With Harvesting Model
}

\author{
Riski Nur Istiqomah Dinnullah', Trija Fayeldi² \\ 1,2 Department of Mathematics Education, Kanjuruhan University \\ Email: ky2_zahra@unikama.ac.id,trija_fayeldi@unikama.ac.id
}

\begin{abstract}
Recently, exploitation of biological resources and the harvesting of two populations or more are widely practiced, such as fishery or foresty. The simplest way to describe the interaction of two species is by using predator prey model, that is one species feeds on another. The Leslie-Gower predator prey model has been studied in many works. In this paper, we use Euler method to discretisize the modified Leslie-Gower with harvesting model. The model consists of two simultanious predator prey equations. We show numerically that this discrete numerical scheme model is dynamically consistent with its continuous model only for relatively small step-size. By using computer simulation software, we show that equlibrium points can be stable, saddles, and unstable. It is shown that the numerical simulations not only illustrate the results, but also show the rich dynamics behaviors of the discrete system.
\end{abstract}

Keywords: Leslie-Gower; Euler method; rich dynamics; dynamically consistent

\section{INTRODUCTION}

For many years, the predator-prey relationship has been the most popular topics in mathematical modelling. One of the model is known as Leslie-Gower predator-prey model. It was introduced by [1] and written as follow.

$$
\begin{aligned}
& \frac{d x}{d t}=\left(r_{1}-a_{1} x\right) x-p(x) y \\
& \frac{d y}{d t}=\left(r_{2}-\frac{a_{2} y}{x}\right) y
\end{aligned}
$$

where $x(t)$ represents the density of the prey at time $t$ with intrinsic growth rate $r_{1}$; while $y(t)$ represents the density of the predator at time $t$ with intrinsic growth rate $r_{2} . a_{1}$ and $a_{2}$ are the strength of competition among individuals of the prey and predator respectively. The main difference between Leslie-Gower model and Lotka-Volterra that is in Leslie-Gower model carrying capacity of the predator is proportional to the number of prey [2]. Since then, the model has been modified by numerous experts, such as [3] and [4].

Finite difference has been used to discretize the continous model to find its numerical solution approximation. The common method is Euler Method [5]. This technique has been applied to various models, for instance [6], [7] , [8] , and [9]. It is found that the obtained discrete model may show rich dynamics. However, divergent approximation may be occur. Hence, stepsize plays an important role to avoid inconsistency of the model. 


\section{METHODS}

\section{Numerical Discretization and Its Equilibria}

The model we use in this paper is written as follow.

$$
\begin{aligned}
& \frac{d H}{d t}=\left(r_{1}-a_{1} P-b_{1} H\right) H-c_{1} H \\
& \frac{d P}{d t}=\left(r_{2}-\frac{a_{2} P}{1+H}\right) P-c_{2} P
\end{aligned}
$$

where $H$ and $P$ represent the density of the prey and predator in time t respectively. In this model, we also assume that $0<c_{i}<r_{i}, i=1,2$ to control the density of the prey and predator.

We are now derive the discrete version of the (2). Applying Euler scheme to the first equation of (2) leads us to the following form:

$$
\begin{gathered}
\frac{H_{n+1}-H_{n}}{h}=\left(r_{1}-a_{1} P_{n}-b_{1} H_{n}\right) H_{n}-c_{1} H_{n} \\
H_{n+1}-H_{n}=h\left[\left(r_{1}-a_{1} P_{n}-b_{1} H_{n}\right) H_{n}-c_{1} H_{n}\right] \\
H_{n+1}=H_{n}+h\left[\left(r_{1}-a_{1} P_{n}-b_{1} H_{n}\right) H_{n}-c_{1} H_{n}\right] \\
H_{n+1}=H_{n}+h H_{n}\left[\left(r_{1}-a_{1} P_{n}-b_{1} H_{n}\right) H_{n}-c_{1}\right]
\end{gathered}
$$

Applying Euler scheme to the second equation of (2) leads us to the following form:

$$
\begin{gathered}
\frac{P_{n+1}-P_{n}}{h}=\left(r_{2}-\frac{a_{2} P_{n}}{1+H_{n}}\right) P_{n}-c_{2} P_{n} \\
P_{n+1}-P_{n}=h\left[\left(r_{2}-\frac{a_{2} P_{n}}{1+H_{n}}\right) P_{n}-c_{2} P_{n}\right] \\
P_{n+1}=P_{n}+h\left[\left(r_{2}-\frac{a_{2} P_{n}}{1+H_{n}}\right) P_{n}-c_{2} P_{n}\right] \\
P_{n+1}=P_{n}+h P_{n}\left[\left(r_{2}-\frac{a_{2} P_{n}}{1+H_{n}}\right)-c_{2}\right]
\end{gathered}
$$

Combining (3) and (4) leads us to the following system:

$$
\begin{aligned}
& H_{n+1}=H_{n}+h H_{n}\left[\left(r_{1}-a_{1} P_{n}-b_{1} H_{n}\right) H_{n}-c_{1}\right] \\
& P_{n+1}=P_{n}+h P_{n}\left[\left(r_{2}-\frac{a_{2} P_{n}}{1+H_{n}}\right)-c_{2}\right]
\end{aligned}
$$

Having found the discrete version, the next step is find the equilibria of (5). Suppose that $\left(H^{*}, P^{*}\right)$ is the equilibrium of $(5)$, then we have

$$
\begin{aligned}
& H^{*}=H^{*}+h H^{*}\left(\left(r_{1}-a_{1} P^{*}-b_{1} H^{*}\right)-c_{1}\right) \\
& 0=h H^{*}\left[\left(r_{1}-a_{1} P^{*}-b_{1} H^{*}\right)-c_{1}\right]
\end{aligned}
$$

Since step-size $h>0$ then we have

$$
0=H^{*}\left(\left(r_{1}-a_{1} P^{*}-b_{1} H^{*}\right)-c_{1}\right)
$$

that is either

$$
H^{*}=0
$$


or

$$
\begin{aligned}
0 & =\left(r_{1}-a_{1} P^{*}-b_{1} H^{*}\right)-c_{1} \\
b_{1} H^{*} & =r_{1}-a_{1} P^{*}-c_{1} \\
H^{*} & =\frac{r_{1}-a_{1} P^{*}-c_{1}}{b_{1}}
\end{aligned}
$$

On the other hand we have

$$
\begin{array}{r}
P^{*}=P^{*}+h P^{*}\left[\left(r_{2}-\frac{a_{2} P^{*}}{1+H^{*}}\right)-c_{2}\right] \\
0=h P^{*}\left[\left(r_{2}-\frac{a_{2} P^{*}}{1+H^{*}}\right)-c_{2}\right]
\end{array}
$$

since $h>0$ then we have

$$
0=P^{*}\left[\left(r_{2}-\frac{a_{2} P^{*}}{1+H^{*}}\right)-c_{2}\right]
$$

that is either

$$
P^{*}=0
$$

or

$$
\begin{aligned}
0 & =r_{2}-\frac{a_{2} P^{*}}{1+H^{*}}-c_{2} \\
\frac{a_{2} P^{*}}{1+H^{*}} & =r_{2}-c_{2} \\
P^{*} & =\frac{r_{2}-c_{2}}{a_{2}}\left(1+H^{*}\right)
\end{aligned}
$$

By looking at (8) and (12), we have the first equilibrium, that is

$$
E_{1}=(0,0)
$$

Next, by substituting (8) to (13) we have the second equilibrium, that is

$$
E_{2}=\left(0, \frac{r_{2}-c_{2}}{a_{2}}\right)
$$

The third equilibrium is found by substituting (12) to (9), that is

$$
E_{3}=\left(\frac{r_{1}-c_{1}}{b_{1}}, 0\right)
$$

The last equilibrium is found by solving (9) and (13), that is

$$
E_{4}=\left(\frac{k_{1}-k_{2}}{1+k_{2}}, \frac{b_{1}}{a_{1}}\left(1+\frac{k_{1}-k_{2}}{1+k_{2}}\right) k_{2}\right)
$$

where $k_{1}=\frac{r_{1}-c_{1}}{b_{1}}$ and $k_{2}=\frac{r_{2}-c_{2}}{a_{2}}$. 


\section{RESULTS AND DISCUSSION}

\section{Numerical Simulation}

In this section, we apply the model along with its numerical simulation. For our purpose, we use numerical simulation software, i.e Matlab. For the first simulation, we use the following parameters: $r_{1}=0.5 ; c_{1}=0.3 ; r_{2}=0.4 ; c_{2}=0.1 ; a_{1}=0.1 ; a_{2}=0.1 ;$ and $b_{1}=0.05$.

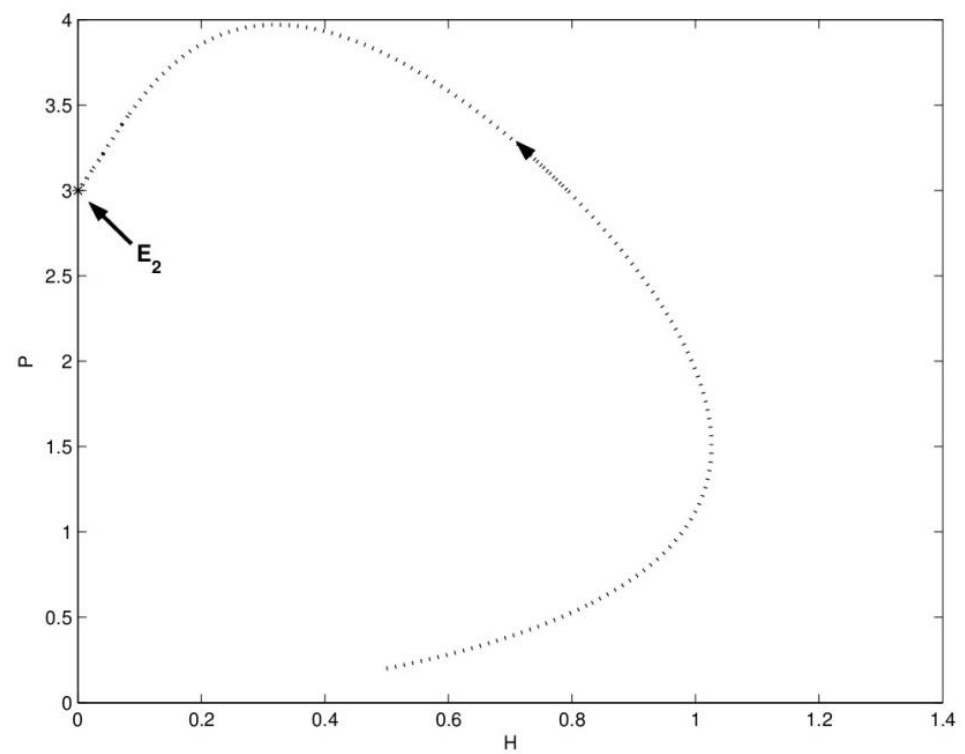

Figure 1. Numerical Solution Approach $E_{2}$

In Figure 1 , we use $(0.5 ; 0.2)$ as the initial condition and step-size $h=0.1$. We found that the numerical solution approach the $E_{2}=(0,3)$. From this simulation, we can infer that the equilibrium point $E_{2}$ is stable for relatively small step-size.

For the second simulation, we use parameters as follow: $r_{1}=0.8 ; c_{1}=0.1 ; r_{2}=$ $0.6 ; c_{2}=0.5 ; a_{1}=0.5 ; a_{2}=0.8$; and $b_{1}=0.8$.

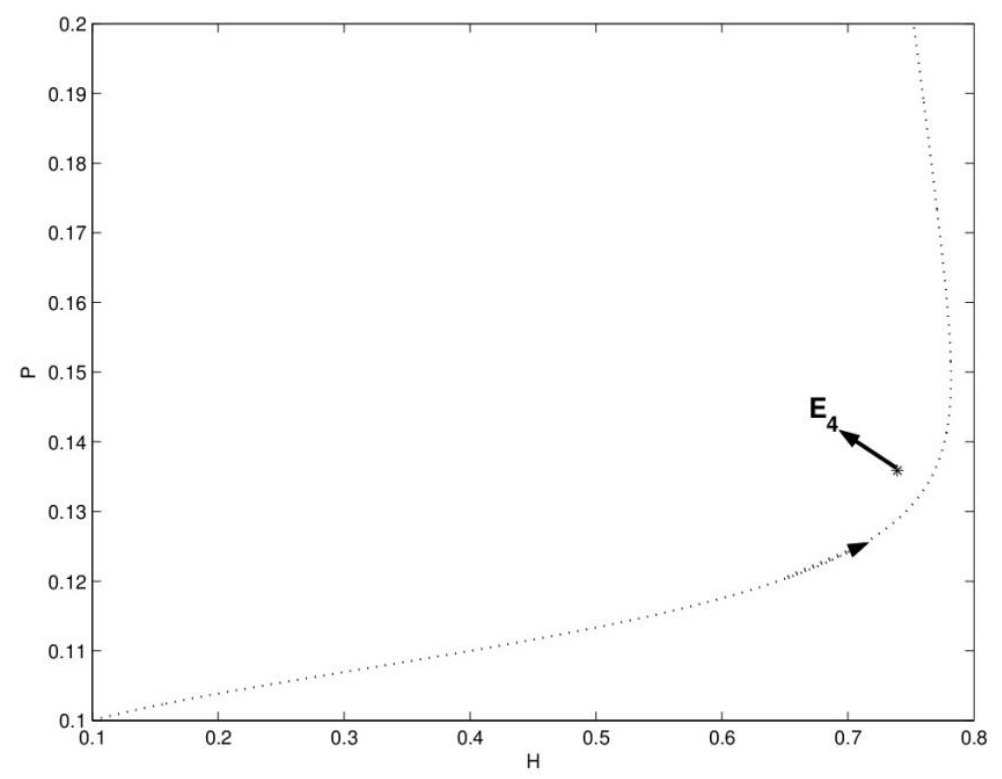

Figure 2. Numerical Solution of Second Simulation

In Figure 2 , we use $(0.1 ; 0.1)$ as the initial condition. We found that the numerical solution is close to $E_{4}$ but never reach the point. From the simulation, we can conclude that $E_{4}$ is an unstable equilibrium. 
In the third simulation, we use parameters as follow: $r_{1}=0.8 ; c_{1}=0.1 ; r_{2}=0.4 ; c_{2}=$ $0.1, ; a_{1}=0.9 ; a_{2}=0.2$; and $b_{1}=0.1$.

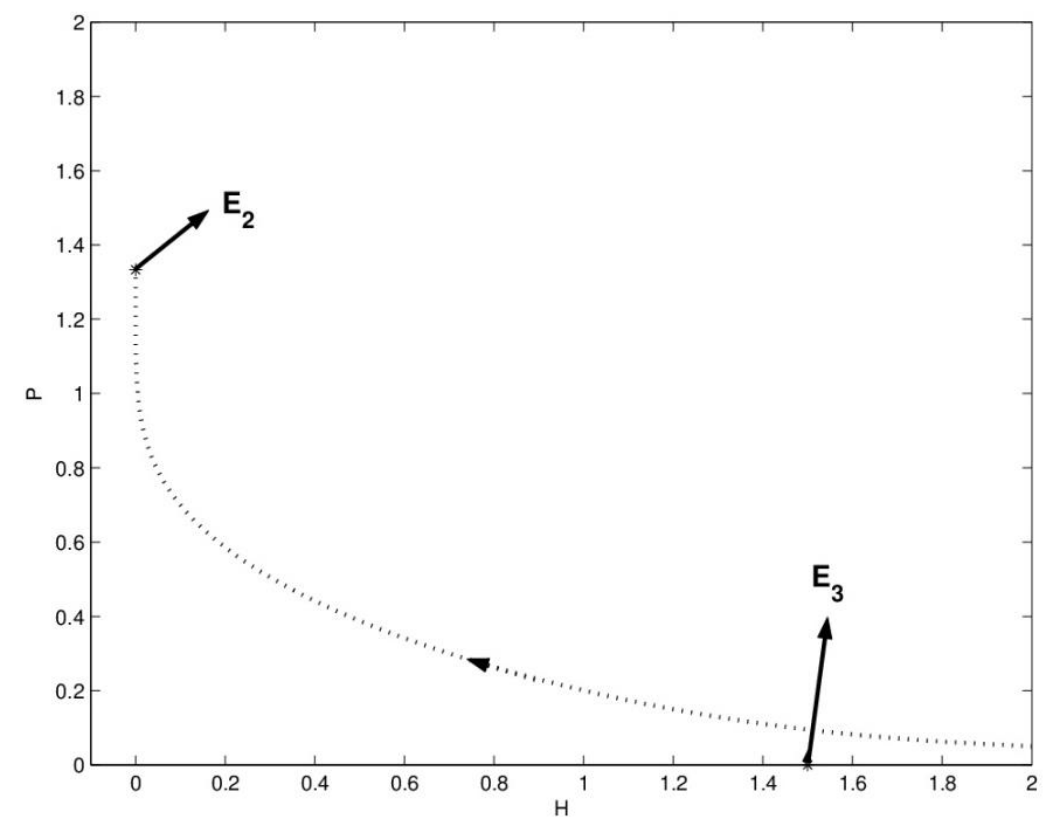

Figure 3. Numerical Solution of Third Simulation

In Figure 3 , we use $(0.01 ; 0.01)$ as initial condition. We found numerically that $E_{1}$ is an unstable equilibrium while $E_{2}$ is a stable equilibrium.

Finally, for the last simulation, we use parameters as follow: $r_{1}=0.5 ; c_{1}=0.2 ; r_{2}=$ $0.9 ; c_{2}=0.5, ; a_{1}=1.8 ; a_{2}=0.3 ;$ and $b_{1}=0.2$.

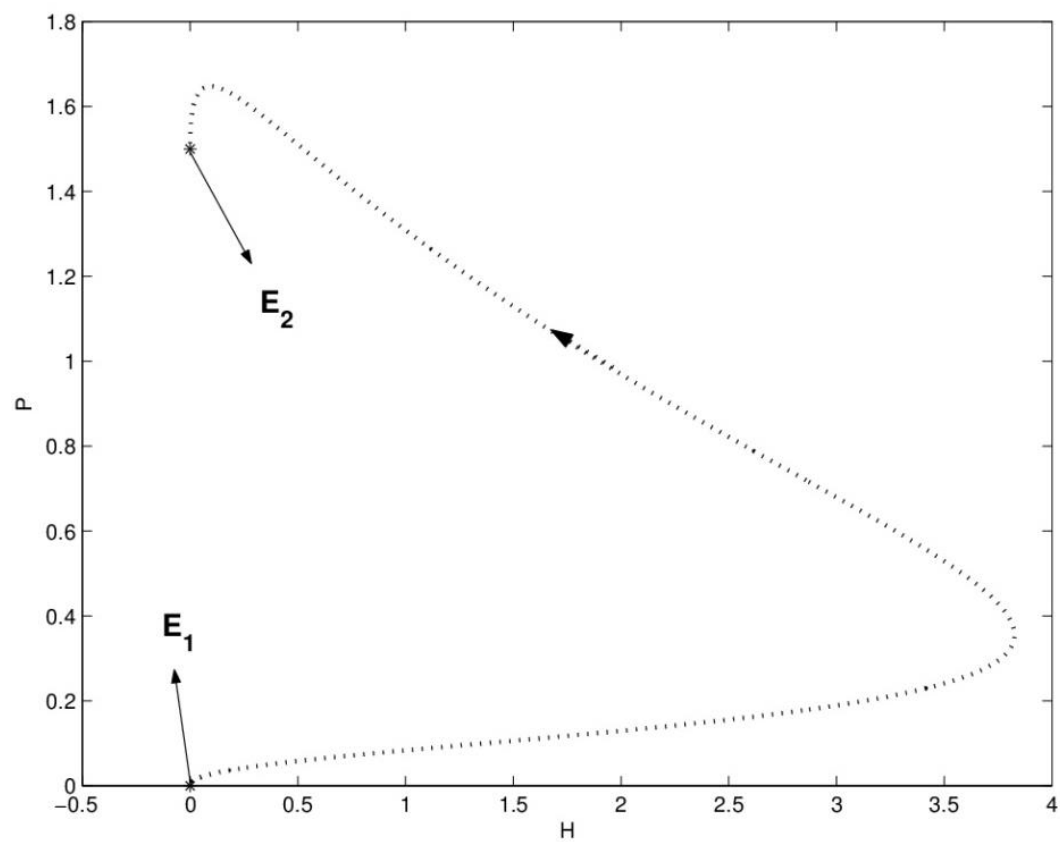

Figure 4. Numerical Solution of The Fourth Simulation

In Figure 4, we use $(2 ; 0.5)$ as the initial condition. From numerical simulation, we found that the solution close to $E_{3}$ but at the end of computation reach the $E_{2}$ equilibrium. So we can conclude that $E_{3}$ is an unstable equilibrium. 


\section{CONCLUSION}

From this paper, a discrete numerical scheme of modified Leslie-Gower with harvesting model has been investigated. We use Euler-method to discretisize the model. We found four equilibrum points with their properties. The $E_{1}, E_{3}$, and $E_{4}$ are unstable equilibrium, while $E_{2}$ is the stable one. It is shown that the discrete model is dynamically consistent with its continuous model only for relatively small step-size $h$. Further works, especially about stability-analysis need to be considered. Furthermore, the discrete model may show complex dynamical behaviors, such as bifurcation and chaos phenomenon.

\section{REFERENCES}

[1] P.H. Leslie, "Some Further Notes on The Use of Matrices in Population Mathematics," Biometrika, vol. 35, pp. 213-245, 1948.

[2] Q. Yue, "Dynamics of a Modified LeslieGower PredatorPrey Model with Holling-type II Schemes and a Prey Refuge," SpringerPlus, vol. 5, pp. 1-12, 2016.

[3] E. Gonzlez-Olivares and C. Paulo, "A Leslie Gower-type predator prey model with sigmoid functional response," International Journal of Com- puter Mathematics, vol. 92, pp. 18951909, 2015.

[4] A. Kang, Y. Xue, and J. Fu, "Dynamic Behaviors of a Leslie-Gower Ecoepidemiological Model," Discrete Dynamics in Nature and Society, vol. 2015, 2015.

[5] T. Fayeldi, "Skema Numerik Persamaan Leslie Gower Dengan Pemanenan," Cauchy, vol. 3, pp. 214-218, 2015.

[6] P. Das, D. Mukherjee, and A. Sarkar, "Study of an S-I epidemic model with nonlinear incidence rate: discrete and stochastic version," Applied Mathematics and Computation, vol. 218, no. 6, pp. $2509-2515,2011$.

[7] A. E. A. Elsadany, H. A. EL-Metwally, E. M. Elabbasy, and H. N. Agiza, "Chaos and bifurcation of a nonlinear discrete prey-predator system," Computational Ecology and Software, vol. 2, pp. 169-180, 2012.

[8] Z. Hu, Z. Teng, and H. Jiang, "Stability analysis in a class of discrete SIR Sepidemic models," Nonlinear Analysis: Real World Applications, vol. 13, no. 5, pp. 2017 - 2033, 2012.

[9] L. Li, G.Q. Sun, and Z. Jin, "Bifurcation and chaos in an epidemic model withnonlinear incidence rates," Applied Mathematics and Computation, vol. 216, no. 4, pp. 1226 - 1234, 2010. 